\title{
Stem cell transplantation for systemic sclerosis: many steps forward in Poland
}

\author{
Dominique Farge ${ }^{1}$, Aleksandra Zoń-Giebel ${ }^{2}$, \\ Eugeniusz J. Kucharz ${ }^{3}$
}

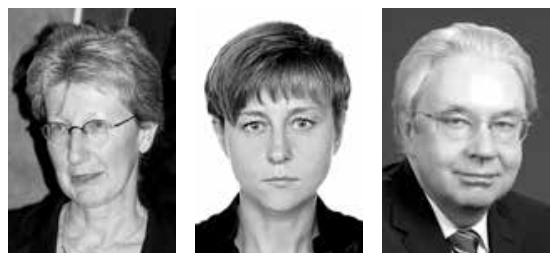

European Society for Blood and Marrow Transplantation (EBMT) Autoimmune Diseases Working Party (ADWP) Chair

${ }^{1}$ Internal Medicine and Vascular Diseases Unit, Hopital St. Louis, Paris, France

${ }^{2}$ Silesian Centre for Rheumatology, Rehabilitation and the Prevention of Disability, Ustroń, Poland

${ }^{3}$ Department of Internal Medicine and Rheumatology, Medical University of Silesia, Katowice, Poland

After twenty years of progress in stem cell transplantation for autoimmune diseases, the final results from the Autologous Stem Cell Transplantation International Scleroderma (ASTIS) trial published a year ago [1] demonstrated that autologous haematopoietic stem cell transplantation (AHSCT) confers better long-term survival than 12 monthly intravenous pulses of cyclophosphamide (Cy) for the treatment of severe or rapidly progressive systemic sclerosis (SSc). In 2015, the Polish Society of Rheumatology, under the Chair of Prof. Eugene J. Kucharz, acknowledged and made efforts to disseminate the important results obtained for treatment of SSc patients. Indeed, many Polish rheumatologists and their voluntary patients as well as other EULAR (European League Against Rheumatism) colleagues met at the end of February 2015 for the EUSTAR (European Scleroderma Trials and Research Group) meeting. The EUSTAR meeting, hosted in Katowice, contributed to raising awareness about SSc, a rare heterogeneous autoimmune disease, which affects 2-22 people per one million every year and is characterized by vasculopathy, autoantibody formation, low-grade inflammation, and progressive fibrosis in the skin and internal organs (lung, heart, kidney and digestive tract). The limited (IcSSc) and diffuse cutaneous (dcSSc) forms can be distinguished by the extent of skin and organ involvement and autoantibody profile, and rapidly progressing dcSSc within the first 4 years after disease onset, observed in to $20 \%$ of cases, is a life-threatening disease with 3-5 year survival between 50 and $70 \%$.

The EUSTAR Educational Course allowed attendees to gain knowledge in the understanding of SSc's com- plex pathogenesis and the importance of the new 2013 ACR-EULAR criteria [2] for early diagnosis and adequate available therapy. Systemic sclerosis can lead to premature death by heart involvement, lung fibrosis and pulmonary hypertension, especially in patients suffering from dcSSc. These patients constitute up to $30 \%$ of the total SSc population and may benefit from AHSCT. This topic, however, was not addressed during the EUSTAR course. Therefore, a second educational course was organized in Wisla with several Polish and European EBMT members. This second meeting illustrated the activity of Polish rheumatologists in the field of SSc and was a nice opportunity to focus on innovation with stem cell transplantation applied to SSc achieved over the past twenty years.

The rationale for using immunoablation with AHSCT in autoimmune diseases is based on experimental studies, results from early phase I-II studies and several retrospective registry studies [3-7]. Today, more than 4000 patients worldwide (EBMT, CIBMTR, and an Asian registry) have received an AHSCT for an AD alone, and in the EBMT registry, 2000 patients have been recorded until November 2015. The most common indications are multiple sclerosis (MS) and SSc. Autologous haematopoietic stem cell transplantation for these diagnoses was performed in more than 245 transplant centres in 39 countries, including 81 cases in Poland, among them 17 for SSc. The mechanism of action in SSc has been better elucidated, and a few studies have shown initial immunosuppression with evidence of subsequent thymic reprocessing or increased regulatory $\mathrm{T}$-cell activity in all types of AD [7] and the capacity to reset the immune response.

Address for correspondence:

Prof. Dominique Farge, Internal Medicine and Vascular Diseases Unit, Hopital St. Louis, Paris, INSERM 1160 Unit Paris 7 Diderot University Sorbonne Paris Cite 1, France, e-mail: dominique.farge-bancel@aphp.fr

Submitted: 26.11.2015; Accepted: 30.12.2015 
In the most severe form, dcSSc, registry analyses have demonstrated that AHSCT induced a major regression of SSc dermal fibrosis, confirmed by histological analysis, which had never been previously reported with any other treatment in SSc. Prolonged follow-up of patients up to 7 years confirmed sustained improved functional status, fall in skin score and stabilisation of lung function, whereas death from disease progression was significantly lower compared to the 5-year mortality rate estimated at 30\% in such severe SSc patients. These results were the basis for the ASTIS trial [1] comparing AHSCT [cyclophosphamide (antithymocyte globulin (ATG) and CD34+ cells selected graft] vs. monthly intravenous pulse of Cy 750 mg/m² for 12 months. Autologous Stem Cell Transplantation International Scleroderma - an international, multi-centre, investigator-based, open label, phase III trial - which has been a unique, groundbreaking academic collaborative project, was coordinated by two leading entities in the field, the EBMT and the EULAR.

From 2001 to 2009, 156 patients from 10 countries with early dcSSc were recruited and followed up until the end of October 2013. The Autologous Stem Cell Transplantation International Scleroderma primary end point was event-free survival, defined as time from randomization until the occurrence of death or persistent major organ failure. Even though AHSCT therapy shows more treatment-related mortality (TRM) in early stages of the follow-up, long-term event-free and overall survival rates are higher in the AHSCT treatment group than in the control group. Other studies elsewhere, such as the US Chicago single centre ASSIST study [8], also elegantly showed that both skin and lung fibrosis regress significantly after AHSCT. Therefore in 2015, according to the revised EBMT guidelines [9, 10], autologous haematopoietic stem cell transplantation should be considered for patients with severe SSc progressing despite standard established and/or approved therapy. Patients should be referred to a centre with JACIE accreditation or equivalent, where appropriate inter-disciplinary interaction with combined haematological and $A D$ specialists allows selection and management of SSc patients. Data reporting and biobanking are central to the development of AHSCT for $A D$ and unique MED-A, and specific MED-B forms for SSc disease can be downloaded at http://www.ebmt.org. Systemic sclerosis patients should be considered for AHSCT when presenting with severe or rapidly progressive disease and disease duration: (a) less than 5 years since onset of first non-Raynaud's symptoms and a modified Rodnan skin score (mRSS) $\geq 15$ plus respiratory (with a DLCO and/or FVC $\leq 70 \%$ of predicted and evidence of interstitial lung disease on HR-CT scan), cardiac (with conduction or rhythm disturbance, pericarditis) or renal involvement with proteinuria > $0.3 \mathrm{~g} / 24$ hours or (b) of 2 years or less and no major organ dysfunction as defined above provided they had an mRSS of at least 20 and an acute phase response (level I). Comprehensive cardiopulmonary screening and pre-transplant evaluation of heart, lung, kidney and gastrointestinal function are critically important to exclude patients at high risk of TRM.

For a long time, knowledge on clinical management and on immunological mechanisms associated with AHSCT for AD has evolved into a very pronounced learning curve, with significant improvements in transplant-related safety and in disease-free survival. In fact, there is a noticeable centre effect among the hundreds of AHSCTs already reported, in which centres more experienced, with larger patient series, report better outcome. This underscores the importance of having educational events and of creating a network of teaching professionals with both experts in $A D$ and in AHSCT aiming to expand AD transplant activity, yet preserving quality of health care.

The success of the Wisla meeting under the auspices of the Polish Rheumatology Society result from common coordinated European efforts, including Poland, where over the past ten years, collaboration between rheumatologists and haematologists has contributed to the development of autologous haematopoietic stem cell transplantation for severe systemic sclerosis patients.

We thank Mrs Manuela Badoglio, Study Coordinator of the Autoimmune Diseases Working Party for her help in updating the ADWP activity.

For any query about ongoing studies or prosla and for reporting HSCT activity, please contact:

manuela.badoglio@upmc.fr at the Paris EBMT office. The authors declare no conflict of interest.

\section{References}

1. van Laar JM, Farge D, Sont JK, et al. Autologous hematopoietic stem cell transplantation vs intravenous pulse cyclophosphamide in diffuse cutaneous systemic sclerosis: a randomized clinical trial. JAMA 2014; 311: 2490-2498.

2. van den Hoogen F, Khanna D, Fransen J, et al. 2013 classification criteria for systemic sclerosis: an American College of Rheumatology/ European League against Rheumatism collaborative initiative. Arthritis Rheum 2013; 65: 2737-2747.

3. Verrecchia F, Laboureau J, Verola O, et al. Skin involvement in scleroderma - where histological and clinical scores meet. Rheumatology (Oxford) 2007; 46: 833-841.

4. Launay D, Marjanovic Z, de Bazelaire C, et al. Autologous hematopoietic stem cell transplant in systemic sclerosis: quantitative high resolution computed tomography of the chest scoring. J Rheumatol 2009; 36: 1460-1463. 
5. Farge D, Labopin M, Tyndall A, et al. Autologous hematopoietic stem cell transplantation for autoimmune diseases: an observational study on 12 years' experience from the European Group for Blood and Marrow Transplantation Working Party on Autoimmune Diseases. Haematologica 2010; 95: 284-292.

6. Nannini C, West CP, Erwin PJ, Matteson EL. Effects of cyclophosphamide on pulmonary function in patients with scleroderma and interstitial lung disease: a systematic review and meta-analysis of randomized controlled trials and observational prospective cohort studies. Arthritis Res Ther 2008; 10: R124.

7. Burt RK, Shah SJ, Dill K, et al. Autologous non-myeloablative haemopoietic stem-cell transplantation compared with pulse cyclophosphamide once per month for systemic sclerosis (ASSIST): an open-label, randomised phase 2 trial. Lancet 2011; 378: 498-506.

8. Farge D, Henegar C, Carmagnat M, et al. Analysis of immune reconstitution after autologous bone marrow transplantation in systemic sclerosis. Arthritis Rheum 2005; 52: 1555-1563.

9. Snowden JA, Saccardi R, Allez M, et al. on behalf of the EBMT Autoimmune Disease (ADWP) and Paediatric Diseases (PDWP) Working Parties. Haematopoietic stem cell transplantation (HSCT) in severe autoimmune diseases: updated guidelines of the European Group for Blood and Marrow transplantation (EBMT). Bone Marrow Transplant 2012; 47: 770-790.

10. Alexander T, Bondanza A, Muraro PA, et al. Stem cell transplantation for severe autoimmune diseases: consensus guidelines of the European Group for Blood and Marrow Transplantation (EBMT) for immune monitoring and biobanking. Bone Marrow Transplant 2015; 50: 173-180.

\section{European Society for Blood and Marrow Transplantation (EBMT)}

The EBMT is a non-profit organisation established in 1974 to allow scientists and physicians to promote transplantation of haematopoietic stem cells and cell therapy including basic and clinical research, education, standardization, quality control, and accreditation. The EBMT develops cooperative studies to save the lives of patients with blood cancers and other life-threatening diseases by advancing the fields of blood and marrow transplantation and cell therapy worldwide through science, education and advocacy. Since 1997, the EBMT Autoimmune Disease Working Party (ADWP) has been dedicated to fostering awareness and clinical collaboration on Stem Cell Transplantation for Autoimmune Diseases. The EBMT ADWP database is the largest collaborative platform in the field, and it has actively contributed to the completion of clinical and research studies for more comprehensive understanding of the best available current therapeutic strategies in autoimmune diseases in rapidly progressive or severe systemic sclerosis, multiple sclerosis, lupus, rheumatoid arthritis and juvenile arthritis, immune cytopenia, inflammatory bowel diseases and other autoimmune diseases, and more recently in early acute insulin dependent diabetes. For further information please visit www.ebmt.org.

Auto-HSCT: Activity per country

ADWP - Number of HSCT: 1826

EBMT Registry - September 2015

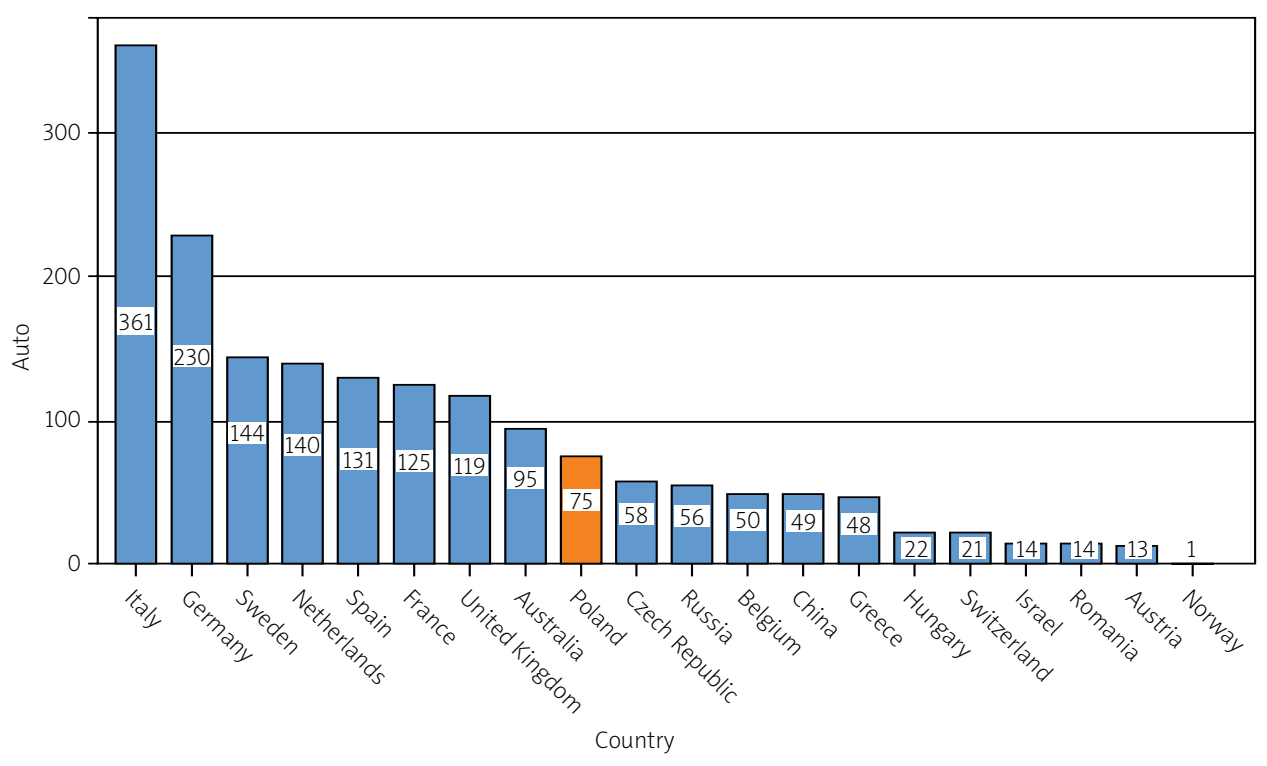




\begin{tabular}{|c|c|c|c|}
\hline EBMT & \multicolumn{3}{|c|}{$\begin{array}{l}\text { ADWP - Number of HSCT: } 1961 \\
\text { EBMT Registry - September } 2015\end{array}$} \\
\hline - MULTIPLE SCLEROSIS & 747 & D HAEMATOLOGICAL & 93 \\
\hline D CONNECTIVE TISSUE & 573 & ITP & 31 \\
\hline SSc & 414 & Evans & 19 \\
\hline SLE & 112 & AlHA & 24 \\
\hline PM-DM & 17 & Other & 19 \\
\hline Sjögren & 3 & - VASCULITIS & 50 \\
\hline Antiphospholipid syndrome & 5 & Wegener's & 12 \\
\hline Other/Unknown & 22 & Behçet's & 9 \\
\hline - ARTHRITIS & 180 & Takayasu & 2 \\
\hline Rheumatoid arthritis & 84 & Polyarteritis nodosa & 4 \\
\hline Juvenile chronic arthritis & & Churg-Strauss & 2 \\
\hline Systemic JIA & 56 & Other/Unknown & 21 \\
\hline Other JIA & 18 & > OTHER NEUROLOGICAL & 83 \\
\hline Polyarticular JIA & 14 & NMO & 21 \\
\hline Psoriatic arthritis & 4 & CIDP & 27 \\
\hline Other & 5 & Myasthenia gravis & 7 \\
\hline - INFLAMMATORY BOWEL & 184 & Other/Unknown & 28 \\
\hline Crohn's disease & 150 & - INSULIN DEPENDENT DIABETES & 20 \\
\hline Ulcerative colitis & 4 & D OTHER/UNKNOWN & 31 \\
\hline Other & 30 & & \\
\hline
\end{tabular}

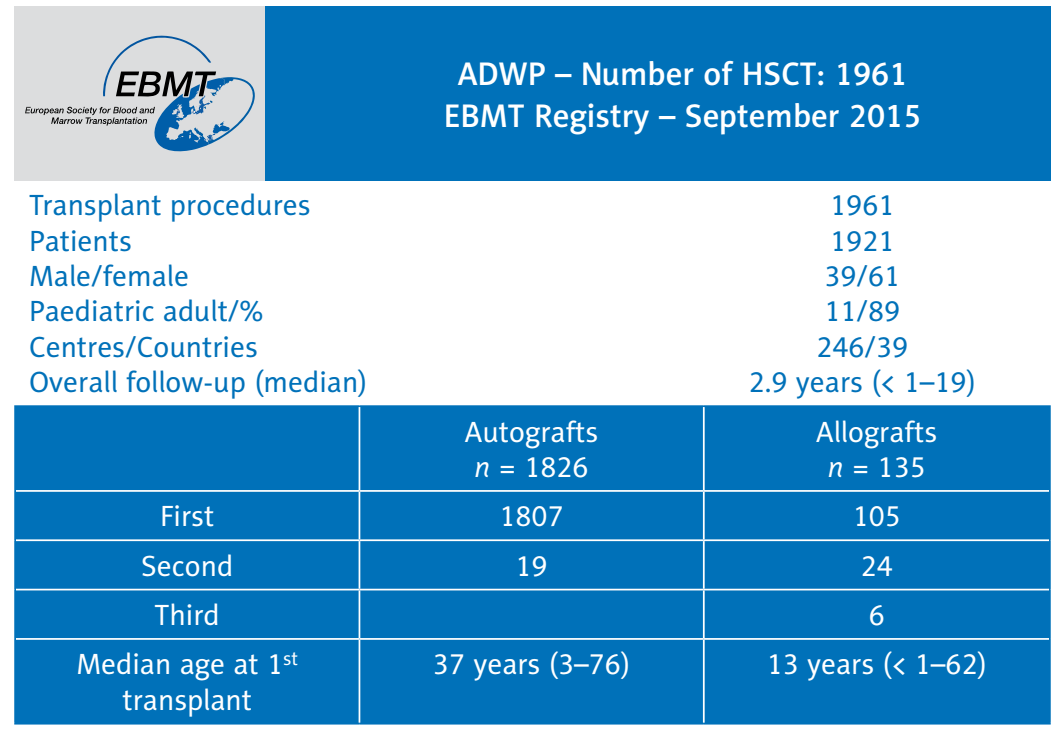

